\title{
Vorwort zur 7. Auflage
}

Das Studienfach Indogermanische Sprachwissenschaft (Kürzel: Indogermanistik) ist seit langem in der Sammlung Göschen (= SG, zuerst Leipzig, dann Berlin) vertreten. Der erste Verfasser eines Bandes „Indogermanische Sprachwissenschaft" war Rudolf Meringer, zur Zeit der 1. Auflage k.k.ao. Professor an der Universität Wien, z.Z. der 3. Auflage k.k.o.ö. Professor an der Universität Graz: 1. Aufl. 1897 (= SG Nr. 59) 136 S. (I. Hauptstück. Die Lehre von der Sprache und ihren Veränderungen; II. Hauptstück. Die indogermanischen Sprachen; III. Hauptstück. Die indogermanische Grundsprache [mit Betonung, Lautlehre und Formenlehre]; IV. Kultur und Urheimat der Indogermanen; p. 1-66 noch in Fraktur gesetzt); 2., durchgesehene Aufl. 1899 (= SG Nr. 59) 136 S.; 3., durchges. Aufl. 1903 (= SG Nr. 59) 151 S. (im II. Hauptstück kommt der Abschnitt $n$. [Auslautgesetze] dazu; Kapitel IV. gilt jetzt ebenfalls als Hauptstück; neu ist ferner am Werkende die Erklärung der Abkürzungen).

Kurz nach dem 2. Weltkrieg konnte Hans Krahe als neuer Autor gewonnen werden, z.Z. der 1. Auflage a.o. Professor an der Universität Würzburg, z.Z. der 2. Aufl. o.ö. Professor an der Universität Heidelberg, seit der 3. Auflage o.ö. Professor an der Universität Tübingen: 1. Aufl. 1943 (= SG 59) 134 S. (I. Teil: Der indogermanische Sprachstamm; II. Teil: Lautlehre; III. Teil: Formenlehre); 2. Aufl. 1948 (= SG 59) 134 S. (keine Veränderungen); 3. Aufl. neu in zwei Bänden bearbeitet: I. Einleitung und Lautlehre 1958 (= SG 59) 106 S. (I. Teil: Allgemeines; II. Teil: Lautlehre) und II. Formenlehre 1959 (= SG 64) 100 S.; 4. Aufl. überarbeitet (I) bzw. neu bearbeitet (II): I. Einleitung und Lautlehre 1962 (= SG 59) 110 S. und II. Formenlehre 1963 (= SG 64) 100 S.; 5. Aufl. I 1966 (= SG 59) (keine Veränderungen) und II 1969 (= SG 64) (keine Veränderungen); 6. Aufl. unverändert 1985 (die Bände I und II sind unter der neuen Nummer SG 2227 in einem Band vereinigt).

Der Band von Krahe ist über fünfzig Jahre alt. Die 6. Auflage zeigt sich zwar äußerlich im neuen Gewand der Sammlung Göschen, innerlich ist sie aber im wesentlichen ein Kind der 3. Auflage aus den sechziger 
Jahren geblieben. Kennzeichnend für die damalige Forschungslage ist die Bemerkung zur „Laryngaltheorie“ p. 101: „Die in jüngster Zeit von einer Reihe von Indogermanisten vertretene sog. „Laryngaltheorie“, nach welcher der idg. Grundsprache außer den hier ... verzeichneten Lauten noch gewisse Laryngale (Kehlkopflaute) bzw. weitere Murmelvokale zugeschrieben werden, ist in dem vorliegenden Büchlein unberücksichtigt geblieben. Der Verf. ist der Ansicht, daß in einer Darstellung, welche in erster Linie für Studierende und zur Einführung in ein Fachgebiet bestimmt ist, nur möglichst gesicherte Forschungsergebnisse dargeboten werden sollten. Die „Laryngaltheorie“ kann aber weder in ihrer Substanz noch in ihrer Methodik als gesichert gelten." Dem letzten Satz ist entschieden zu widersprechen: Der in der Indogermanistik gebräuchliche Ausdruck „Laryngaltheorie“ ist historisch bedingt, er ist aber irrefuhrend und darf nicht der Idee Raum geben, die Laryngale seien graue Theorie. Die Laryngale gehören heute zum gesicherten Lautbestand des Urindogermanischen, s.u. L $314 \mathrm{ff}$. Auf Schritt und Tritt finden sich im Text von Krahe Angaben oder Rekonstrukte, über die wir heute mehr wissen oder für die wir inzwischen adäquatere Einsichten gewonnen haben.

Mitte der achtziger Jahre konnte der Verlag Heiner Eichner und Manfred Mayrhofer dafür gewinnen, für die Reihe Göschen eine neue „Indogermanische Sprachwissenschaft“ zu schreiben. M. Mayrhofer verfaßte dafür bereits 1985 den Teil Lautlehre, H. Eichner wollte sich um Einleitung und Formenlehre kümmern. 1988 wurde der Band öffentlich angekündigt. Eine Publikation ist aber nie erfolgt. Schwierigkeiten türmten sich nämlich auf der Seite von H. Eichner auf. Sein Manuskript war zwar inzwischen recht gut gediehen, die zeitlichen Vorgaben, die räumliche Enge der ihm insgesamt zur Verfügung stehenden rund zweihundert kleinen Göschenseiten und die jedem Autor bekannte Schwierigkeit, den eigenen Namen für etwas nicht wirklich Vollkommenes zu verwenden, hinderten ihn schließlich daran, zu einem Abschluß zu kommen.

Im Dezember 1993 erreichte mich auf Vorschlag von M. Mayrhofer vom Verlag die Anfrage, ob ich den Part von $\mathrm{H}$. Eichner übernehmen würde. Nach Rücksprache mit allen Beteiligten kam 1994 ein Verlagsvertrag zustande. Als Abgabetermin wurde Februar 1996 vereinbart. Meine im Jahr 1995 sich anbahnende Berufung an die Freie Universität Berlin machte die kühnen Zeitpläne alsbald zunichte. Der Wechsel von Hamburg nach Berlin brachte alles andere mit sich, nur nicht die erforderliche Muße für ein Manuskript. Ich bin Brigitte Schöning dankbar, daß sie von der Verlagsseite aus das notwendige Verständnis für meine Zwänge fast nie 
verlor und mir schließlich den jetzigen Termin abgerungen hat. Ich hätte am Text gern weitergewoben, ich muß ihn aber jetzt aus der Hand legen, damit ich den Verleger nicht verliere.

Während ich vom Inhalt her im Herbst 1998 erst die Hälfte des Themas bewältigt hatte, kam eine Umfangsberechnung des bereits vorliegenden Textes im Verlag zum Schluß, das damit der in einem Göschenband zur Verfügung stehende Raum schon weit überschritten war. Als neu aufnehmende Reihe empfahlen sich daher die „de Gruyter Studienbücher“. Sie bieten größere räumliche Freiheit. Ferner verlangen sie keinen bestimmten Satz. Deshalb kann die mit Hilfe von Microsoft Word 7.0 for Windows 95 geschriebene Textvorlage direkt für die Publikation verwendet werden. Fahnenkorrekturen entfallen. Ingeborg Klak hat von der Verlagsseite aus dafür gesorgt, daß die Typographie nicht so altväterisch daherkommt.

Während M. Mayrhofer und H. Eichner in der Nachfolge von H. Krahe nur die Laut- und die Formenlehre darzustellen beabsichtigten, erhalten jetzt zusätzlich auch Syntax und Wortschatz eine eigene Würdigung.

Die Ausführungen zum lautlichen Teil stützen sich auf den Text, den M. Mayrhofer 1985 angefertigt hat. M. Mayrhofer hat mir aber alle Freiheit in der Gestaltung und Formulierung der definitiven Version zugestanden. Für die vorgelegte Version der Lautlehre übernehme ich die alleinige Verantwortung. Matthias Fritz konnte ich erfreulicherweise dafür gewinnen, den syntaktischen Teil zu verfassen. Er hat ferner innerhalb der Einleitung den Abschnitt über die Geschichte der indogermanischen Sprachwissenschaft und die Übersicht über die indogermanischen Sprachen und ihre Quellen mitgestaltet.

M. Fritz, M. Mayrhofer, Elisabeth Rieken, Bernfried Schlerath und Antje Schwinger haben einzelne Abschnitte mit kritischem Auge gelesen und mir ihre Kritik mitgeteilt. Veronika Rittner und Thorsteinn Hjaltason haben einzelne Texte elektronisch erfaßt. Th. Hjaltason und A. Schwinger haben mir bei manchen EDV-Problemen geholfen, Th. Hjaltason speziell bei der Erstellung von Sonderzeichen, A. Schwinger beim Layout. Ihnen allen mein herzliches Dankeschön!

Wie es sich für einen Band in der Sammlung Göschen und für ein „de Gruyter Studienbuch" gehört, soll das Studienbuch eine Einführung in die indogermanische Sprachwissenschaft bringen. Das Studienbuch soll verständlich und informativ über die Probleme und Themenbereiche aus heutiger Sicht referieren. Im Einleitungsteil erlaube ich mir bei den In- 
formationen zum Fach und zum Studium eine Art „Knigge-Stil“. Bei der Darstellung der Fachthematik geht es dagegen objektiv darum, die fragliche Materie in brauchbarer Art und Weise zu präsentieren und zu organisieren und Anreger und Wegweiser für eine Vertiefung zu sein. Die Darstellung erfolgt nach bestem Wissen und Gewissen. Vollständigkeit im Material ist wissenschaftiches Ideal, in einer Einführung hat aber die treffende Wahl von Musterbeispielen und die Beschränkung auf die wichtigste Sekundärliteratur Priorität. Um dies immer wieder deutlich vor Augen zu halten, beginnen die Kapitelüberschriften meist mit einem „zu“. Der Großteil der Beispiele in der Formenlehre, in der Syntax und im Wortschatzteil stammt aus dem Lateinischen, Altgriechischen und Altindischen. Bei der Lautlehre sind die Beispiele dagegen bewußt zahlreicher und stammen aus der gesamten Indogermania, neben der genannten Trias besonders aus dem Hethitischen, Germanischen und Slavischen. Das am Schluß beigefügte Sachregister soll den Benützer unterstützen und ihm zusätzliche Informationen an die Hand geben. Der im Text vertretene Wissensstand ist der von September 1999. Letzte Zusätze werden nach dem Stichtag kurz vor dem definitiven Layout im Dezember eingefügt.

Das hier vorgelegte Studienbuch ist nicht ohne Konkurrenz. Sehr gut ist nach wie vor Rix Hist. Gramm. d. Gr. 1976 (zu den Kurztiteln s.u. die Bibliographie): Vom Griechischen ausgehend ergeben sich treffende Informationen zu allen Belangen der urindogermanischen Laut- und Formenlehre. Die Darstellung wird aber ohne Bezug auf die Fachdiskussion geführt. Aus neuerer und neuester Zeit sind zu nennen: Szemerényi Einführung 4. Auflage 1990, Beekes Introduction 1995, Schmitt-Brandt Indogermanistik 1998 und in gewissem Sinne auch Meiser Hist. Laut- und Formenlehre d. lat. Sprache 1998 (vgl. p. 27-46 das Kapitel „Grundzüge der urindogermanischen Grammatik"). Alle vier Titel haben Vorzüge und Schwächen, und auch bei meinem wird es wohl nicht anders sein. Bei Szemerényi sind die umfassenden Literaturhinweise eine Fundgrube, die Skepsis den Laryngalen gegenüber ist aber störend. Das Buch von Beekes ist illustrativ und gut lesbar, im Bereich von Laut- und Formenlehre stützt er sich aber zu sehr auf nur von ihm selbst und F. Kortlandt vertretene Ansichten. Schmitt-Brandt richtet sich in löblicher Weise an die Anfänger und bemüht sich speziell im lautlichen Bereich um eine breite, die Kreatitivät fördernde Argumentation. Sein Buch kann aber nicht empfohlen werden, weil er den Leser immer wieder in nicht immer deutlich als solche signalisierte Idiosynkrasien führt, die von der Communis opinio abweichen. Meiser behandelt wie Rix Hist. Gramm. d. Gr. 1976 nur die 
Laut- und Formenlehre. Er nimmt in kompetenter, aber notgedrungen knapper (zu knapper) Form Bezug auf die Gegebenheiten der Grundsprache. Von den nicht so seltenen Tippfehlern darf ich aber nicht reden. Wer selbst in einem Glashaus sitzt, ist gut beraten, keine Steine zu werfen.

Ich bin für jedes Feedback dankbar und erbitte es an das Seminar für Vergleichende und Indogermanische Sprachwissenschaft der Freien Universität Berlin (FU), Fabeckstraße 7, D-14195 Berlin-Dahlem. Tel.: 030838-55028; Fax.: 030-838-54207; E-mail: drmeier@zedat.fu-berlin.de; Homepage Internet: http://www.fu-berlin.de/indogermanistik/.

Ich wage den Versuch und richte auf unserer Homepage extra eine Rubrik 'De Gruyter Studienbuch Indogermanische Sprachwissenschaft: Addenda und Corrigenda' ein. Ich nehme mir vor, ab Erscheinen des Buches immer zum 1. Werktag eines neuen Monats die Rubrik mit Addenda und Corrigenda zu ergänzen.

Berlin-Dahlem, am 15. September 1999

Michael Meier-Brügger 
